\title{
Simulation of Noise Propagation Law on Fully Mechanized Mining Face of Coal Mine Based on Finite Element Method
}

\author{
Yuansheng Wang, , Zhiyang Gao ${ }^{2, *}$, Guoxun Jing², Shaoshuai Guo² and Fang Jiang ${ }^{2}$ \\ ${ }^{1}$ School of Aviation, Anyang Institute of Technology, Anyang 455000, China \\ ${ }^{2}$ College of Safety Science and Engineering, Henan Polytechnic University, Jiaozuo 454003, China
}

Received 19 February 2021; Accepted 30 May 2021

\begin{abstract}
The noise pollution on underground fully mechanized mining faces, which is significantly correlated with fatigue, harms the physiological and psychological health of field operation personnel over time. Effectively predicting the noise propagation and attenuation laws of fully mechanized mining faces and mitigating the physical and mental harm caused by a noisy operating environment to personnel has become a research focus. To explore the propagation characteristics of bad noise in a fully mechanized mining face, a coal mine in Henan Province, China was taken as an example, and the noise propagation and attenuation laws at three different excitation positions of sound source (left end, right end, and middle of roadway) were simulated via COMSOL software based on the acoustic theory of long space and the finite element method. Results show that when the excitation point of the sound source is located at two ends of a roadway, the noise declined from $105.7 \mathrm{~dB}$ to $54.5 \mathrm{~dB}$ within the $70-\mathrm{m}$ range from the sound source, with the excitation point being the center. When the excitation point is in the middle, the noise decreased from the initial $105.2 \mathrm{~dB}$ to $51 \mathrm{~dB}$ within the $75-\mathrm{m}$ range from the sound source, with the excitation point being the center. The sound pressure attenuation with the distance of the receiving point in the roadway presents a linear symmetric trend. In the entire roadway space, the sound pressure level at the receiving point attenuates rapidly in the region near the sound source, approximating a linear attenuation. When the receiving point is located at the roadway exit, the sound energy experienced minor attenuation. The conclusions provide a related reference for coal mine enterprises to arrange protective devices for operation personnel and conduct noise control from the perspective of the propagation process of sound energy to reduce the safety accidents caused by human errors from the perspective of ergonomics.
\end{abstract}

Keywords: Fully mechanized mining face of coal mine, COMSOL Multiphysics, Noise field, Propagation characteristics

\section{Introduction}

With the development of modern industry and scientific and technological progress, an increasing number of high-power and high-rotation-speed mechanical equipment have become the main mode of production in the coal mining industry [1]. Owing to the increasing number of mechanical equipment, the narrow working spaces, the strong ventilation directivity, and large equipment loads, a special noise pollution environment is formed in fully mechanized mining faces, thus exerting serious physiological and psychological impacts on the operation personnel. In addition to increasing the incidence rate of occupational diseases, this phenomenon also leads to the unsafe behaviors of operation personnel [2]. Human is a weak link in coal mine safety production. According to data statistics, the total number of coal mining accidents is generally presenting a declining trend, but the production safety accidents caused by human errors and fatigue account for $88.4 \%$ of the total number of accidents on fully mechanized mining faces [3]. Hence, studying the noise propagation and attenuation laws in fully mechanized working faces is an effective path to preventing and reducing the physical and mental harms caused by noise pollution to operating personnel.

However, most of the existing studies concentrated on the noise reduction of mining machinery, namely, physical

*E-mail address: gzy@hpu.edu.cn

ISSN: 1791-2377 @ 2021 School of Science, IHU. All rights reserved.

doi:10.25103/jestr.142.13 noise reduction at the noise source and noise elimination and transformation [4]. Nevertheless, the attenuation and evolution characteristics of the noise propagation path have been scarcely analyzed from the angle of noise propagation laws. In addition, the focus has been mostly on investigation, mechanical noise reduction, and field determination, and the studies on noise control on fully mechanized mining faces from the angles of systems engineering and noise propagation path through man-machine-environment combination and the numerical simulation of coal mines are limited. Hence, the propagation and attenuation laws of noise source in the underground fully mechanized mining face of coal mines were analyzed, the propagation characteristics were identified, and the noise intensity in different areas was measured in this study. The corresponding conclusions provide a scientific basis for coal mine enterprises to formulate prevention and control strategies for occupational diseases and comprehensively reduce underground safety accidents.

\section{State of the Art}

Mechanical noise reduction has always been the focus of most studies with respect to noise control, namely, mechanical noise elimination and transformation of noise source, mainly including passive and active noise reduction. In the aspect of passive noise reduction, through an engine 
decibel experiment on two aircraft, Holmer found that $40 \%$ savings in acoustical material weight could be achieved by installing a damping decoration panel in the engine room, which can effectively reduce the noise in cockpits, kitchens, and bathrooms [5]. Akl studied and developed a new type of intelligent foamed material, established a finite element model under the coupled intelligent foamed plastic and plate chamber system, compared the model prediction results through the vibration and sound pressure level (SPL) feedback control strategies, and verified the noise reduction characteristic of this foamed material [6]. Through the analog computation of the noise map of an important urban road segment, Bunn discovered that the change in pavement or vehicle speed limit is not an effective noise control measure, and comparatively, limiting the passage of heavy vehicles is considered as an effective measure of lowering the noise level [7]. Linus et al. explored the potentials of acoustical metamaterials in the noise control of automobiles and the cabins of armored vehicles and discovered the deficiencies in the aspect of low-frequency noise control [8]. By applying leading-edge sawtooth on the rear airfoil profile, Vemuri experimentally studied the passive noise control of the series NACA 65-710 airfoil profile structure, analyzed the pressure distribution on a static surface and the pressure fluctuation on a moving surface, and revealed the physical phenomenon of the leading-edge tooth and its noise reduction mechanism [9]. Mamontov et al. considered various soundproof board design and calculation procedures to protect houses from the noise in air. The maximum effect could not be achieved by the past procedures. Therefore, they proposed an optimal calculation method for a group of soundproof boards to obtain the optimal value of the objective function [10]. In addition, many predecessors studied noise control systems with respect to active noise reduction, and these systems have been widely applied to various fields. Sun et al. investigated non-Gaussian pulse noise control using the filter-x least mean square (FxLMS) algorithm of the typical active noise control (ANC) and proposed the filter-x least mean $\mathrm{m}$ estimation algorithm based on robust statistics theory [11]. On the basis of the fractional-order signal processing method, Aslam proposed an online auxiliary path model by using the multidirectional fractional step length least square algorithm, improved the adaptive strategy of the secondary online path modeling of the ANC system, and compared narrow-band and wideband noise signals; the comparison results show that the proposed method has a good application effect in the aspects of model precision and convergence rate [12]. By constructing a multiple circular microphone and loudspeaker array system and designing the corresponding space, Sun et al. put forward an ANC algorithm to realize the ANC of the multiple circular array system. The simulation results indicate that the proposed method could achieve an ANC performance equivalent to the conventional spherical array method [13]. To improve the noise reduction performance of an ANC system, Yu applied a global optimization algorithm the quantum particle swarm algorithm to the ANC system, designed a scheme of steady-state path mutation detection and re-convergence processing, and effectively solved the system problem, namely, failure to recover the convergence after the change in the steady-state path [14]. Shakeel et al. proposed a method of solving the saturation nonlinearity of the FxLMS algorithm in the secondary path and verified the advantages of this method under different conditions over the existing methods through the numerical simulation results [15].
For noise control, certain preventive measures have been taken at the end of noise control from the angle of labor protection. Although devices, like hearing protectors, can be used on the fully mechanized mining faces of coal mines to reduce the contact of workers with noise, the success depends on whether the wearers can correctly wear the hearing protectors given that no appropriate training methods on the use of hearing protectors exist in small-scale industries. Therefore, Kim et al. evaluated the protective effect of earplug training on auditory sense and prevented the hearing loss caused by noise [16]. Ryu assessed the application potential of reinforced concrete in lightweight soundproof walls [17]. Latos studied a global noise protection solution for industrial areas based on the concept of virtual sound corridor, obtained an electroacoustic path model through dynamic system identification, and verified the effectiveness and practicality of this system in hearing protection according to the noise test records of the Rybnik power plant [18]. Butorina analyzed the factors influencing the noise emission of rail tracks, proposed an estimation method for the noise level of railway traffic flow, and found that the effective measures of reducing train noise are a set of measures that not only need to reduce the noise sources but also mitigate the noise on the propagation path, namely, the acoustic screen [19]. Susanto et al. explored the noise plotting of the mechanized mineral processing industry to evaluate the noise exposure level of workers and found that the highest noise level generated in industrial areas is within 88-97 dBA, and the study is of great importance to noise protection and management [20]. Anatoliy proposed an acoustic panel absorber with a high hygienic property to reduce noise, improve agricultural production conditions, and strengthen the labor protection of workers [21]. Holt analyzed noise-induced damage and ear protection models with rats as study objects from the perspective of the pathogenesis of noise [22].

In summary, noise control has been investigated by predecessors mainly from the angles of noise source and receiver, and mechanical noise reduction has been the focus in which the passive noise reduction from the angle of materials science is the main implementation plan. However, few studies have proposed protective measures by combining the noise propagation characteristics based on the analysis of noise propagation laws. In addition, predecessors mainly adopted physical experiments and the control algorithm, while the evolution laws and attenuation features during the noise propagation process is not revealed.

Therefore, to address the deficiencies of existing studies, the actual environmental characteristics of fully mechanized mining faces were combined in this study. Interdisciplinary theories, such as safety engineering, environmentology, computer science, and man-machine interaction, were used to comprehensively analyze the noise propagation laws and attenuation features of the underground fully mechanized mining faces of coal mines. Furthermore, the sound field in roadways was numerically simulated via COMSOL to identify the noise evolution characteristics in different areas, expecting to provide related reference for alleviating the physical and mental harm caused by noise to operation personnel, enhance the behavioral reliability, and reduce human errors in coal mines.

The remainder of this study is organized as follows. Section 3 expounds on the implementation process of the finite element method (FEM) and the establishment of a simulation model. Section 4 presents the simulation result analysis and discussion on the underground noise 
propagation laws by taking a coal mine in Henan Province, China as an example. Section 5 draws the related conclusions obtained from this study.

\section{Methodology}

Bin proposed the essence of the acoustic problem, namely, wave equation [23], marking the birth of modern acoustics. Subsequently, the breakthrough in computational acoustics boosted the rapid development of modern acoustics. As simulation software that integrates preprocessing, solving, and postprocessing, COMSOL Multiphysics applies to the simulation analysis of physical phenomena described by a partial differential equation and the dynamic simulation of noise propagation in the noise field. On this basis, this simulation software was used in this study to explore the noise distribution laws and attenuation characteristics in different areas within the roadway of a fully mechanized mining face. The accuracy of the method was verified by combining measured data with the simulation results to comprehensively protect underground operators in coal mines from noise pollution and strengthen the safety management ability of coal mining work. The specific solution idea of the finite element simulation is shown in Figure 1.

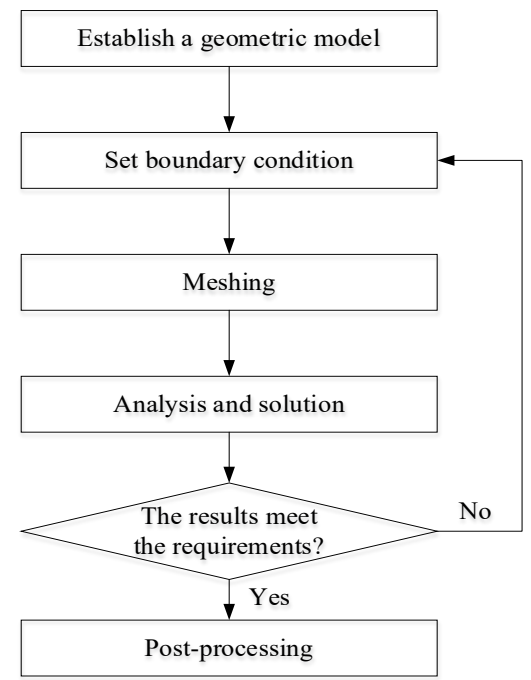

Fig.1. Flow chart of finite element simulation

\subsection{Principle of software model}

The airflow in underground fully mechanized mining faces is a very complicated fluid flow problem, which is dominated by three basic equations: the equations of continuity, motion, and state. The wave equation reflecting acoustical problems can be inferred by the three basic equations. For a $3 \mathrm{D}$ sound wave, the three equations are expressed as Equations (1), (2), and (3), respectively:

$$
\frac{\partial \rho}{\partial t}+\frac{\partial(\rho u)}{\partial x}+\frac{\partial(\rho v)}{\partial y}+\frac{\partial(\rho w)}{\partial z}=0
$$

$\rho\left(\frac{\partial \vec{V}}{\partial t}+(\vec{V} \cdot \nabla) \vec{V}\right)+\nabla p=0$

$$
\frac{\Delta p}{P_{0}}=\gamma \frac{\Delta \rho}{\rho_{0}}
$$

where $\rho$ is the density; $u, v$, and $w$ are the components of the velocity vector in directions $x, y$, and $z$, respectively; $\vec{V} \cdot \nabla$ is the undirected operator, and $\vec{V} \cdot \nabla=u \frac{\partial}{\partial x}+\frac{\partial}{\partial y}+\frac{\partial}{\partial z} ; \nabla p$ is the operation of the $\nabla$ operator for scalar quantity $\mathrm{p}$, and $\nabla p=\vec{i} \frac{\partial p}{\partial x}+\vec{j} \frac{\partial p}{\partial y}+\vec{k} \frac{\partial p}{\partial z} ; P_{0}$ and $\rho_{0}$ represent the static pressure and density of air, respectively; $\Delta p$ and $\Delta \rho$ denote the increments of SPL and density, respectively; and $\gamma$ is the specific heat ratio of air and generally taken as $\gamma=4.1$ for air.

The wave equation in the $3 \mathrm{D}$ sound wave was inferred from the equations of continuity, motion, and state in fluid medium as follows:

$$
\frac{\partial^{2} \Phi}{\partial t^{2}}+\frac{\partial}{\partial t}(\nabla \Phi)^{2}+\frac{1}{2} \nabla \Phi \cdot \nabla(\nabla \Phi)^{2}=c^{2} \nabla^{2} \Phi
$$

where $\Phi$ is the scalar potential, and $\nabla \Phi=\vec{i} u+\vec{j} v+\vec{k} w ; c$ is the sound velocity in fluid medium; and $\nabla^{2}$ is the Laplace operator, and $\nabla^{2}=\frac{\partial^{2}}{\partial x^{2}}+\frac{\partial^{2}}{\partial y^{2}}+\frac{\partial^{2}}{\partial z^{2}}$.

\subsection{Physical model}

The roadway structure chart of fully mechanized coal mining shows the following roadway features. (1) The roadway shape in the axial direction was unchanged, and its cross-section is generally trapezoidal or rectangular. (2) The roadway space is long with four faces enclosed (at the two ends are the intake and return airways), where the 1D length is larger than the other 2D lengths by several times (see Figure 2). Therefore, the roadway in the fully mechanized mining face could be simulated as a structural model of a narrow and long acoustic space. As shown in the Figure 2, $l$, $m$, and $n$ are the length, width, and height of the roadway, respectively $(1>\mathrm{m}, 1>\mathrm{n})$.

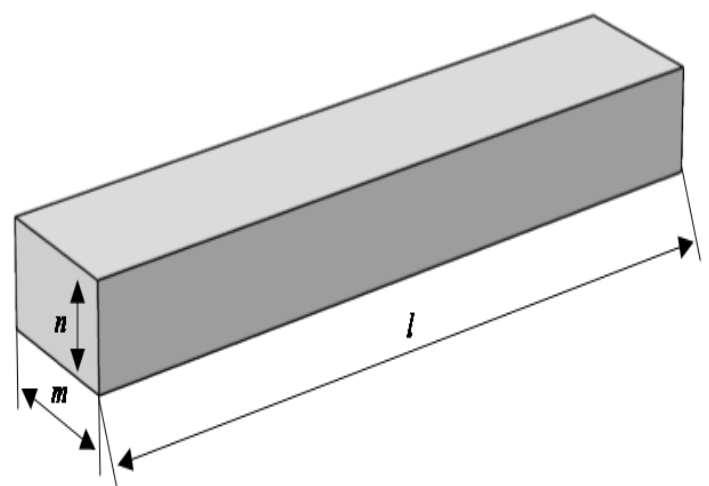

Fig.2. Schematic diagram of roadway in fully mechanized working face

The fully mechanized mining face has a wide range of sound source types of environmental noise. The noise attenuation laws in the roadway were analyzed in this study by using a single sound source-coal cutter and solved through the equation of acoustic diffusion in the acoustic module of COMSOL Multiphysics. This equation can be 
applied in solving the diffusion problem of sound energy density and the corresponding attenuation curve of sound energy. The roadway material domain is set as the air, with a density of $\rho=1.29 \mathrm{~kg} / \mathrm{m} 3$ and a sound velocity of $c=343$ $\mathrm{m} / \mathrm{s}$. Given that the length is much greater than the width and the height, the sound field of this long space is not diffused. The energy density distribution of noise in the space is simulated by setting the exciting power of the noise source as $Q=1 \mathrm{~W}$, and the model is finally solved.

\subsection{Simulation method}

In this study, the noise propagation was simulated by taking the fully mechanized mining face of Shenhuo Coal Mine in Shangqiu City, Henan Province, China as an example. The specific data for the roadway calculation model for this fully mechanized mining face are as follows: $L=150 \mathrm{~m}$ (length), $M=5 \mathrm{~m}$, and $N=3 \mathrm{~m}$ (height); and the acoustic absorption coefficients of the goaf, the rock, the coal wall, and the steel plate of the machines were set according to Table 1 . The acoustic absorption coefficient was set to 1 at the two ends to simulate the states under no sound reflection, the exciting power was $1 \mathrm{~W}$, the sound source was localized at the leftmost end of the roadway goaf in the fully mechanized coal mining, and the receiving point was arranged at the axial position of the sound source along the roadway center.

Table 1. Sound absorption coefficient of fully mechanized roadway

\begin{tabular}{c|c}
\hline Region & Sound absorption coefficient \\
\hline Goaf & 0.8 \\
Rock & 0.25 \\
Coal wall & 0.4 \\
Machine steel plate & 0.25 \\
\hline
\end{tabular}

\section{Results Analysis and Discussion}

4.1 Impact analysis of distance of receiving point in roadway on noise attenuation

Through the above layout of the simulation process and the simulation results of COMSOL, the attenuation curves of the SPL with the increasing distance of the receiving point from the sound source were drawn as shown in Figure 3. Figure 3 indicates that the SPL attenuated rapidly (from $105.7 \mathrm{~dB}$ to $54.4 \mathrm{~dB}$ ) within the $70-\mathrm{m}$ range from the receiving point to the sound source in the entire roadway space, with the attenuation ratio reaching as high as $48.5 \%$, basically presenting a linear attenuation. As the receiving point was localized at over $10 \mathrm{~m}$ from the roadway exit toward the roadway exit, the sound energy was basically unchanged in the roadway, and minor attenuation amplitude reappeared when the receiving point was near the exit of the end face.

When the sound source was localized in the middle of the roadway goaf in the fully mechanized coal mining and the receiving point at the axial position of sound source along the roadway center, the attenuation curves of the SPL with the increasing distance from the receiving point to the sound source are displayed in Figure 4. The SPL was the maximum at the exciting position and declined from the initial $105.2 \mathrm{~dB}$ to $51 \mathrm{~dB}$ (attenuation ratio: $51.5 \%$ ) within the $75-\mathrm{m}$ range from the sound source with the center as the exciting point. The SPL attenuation with the distance of the receiving point from the sound source in the roadway showed a linear symmetric trend.

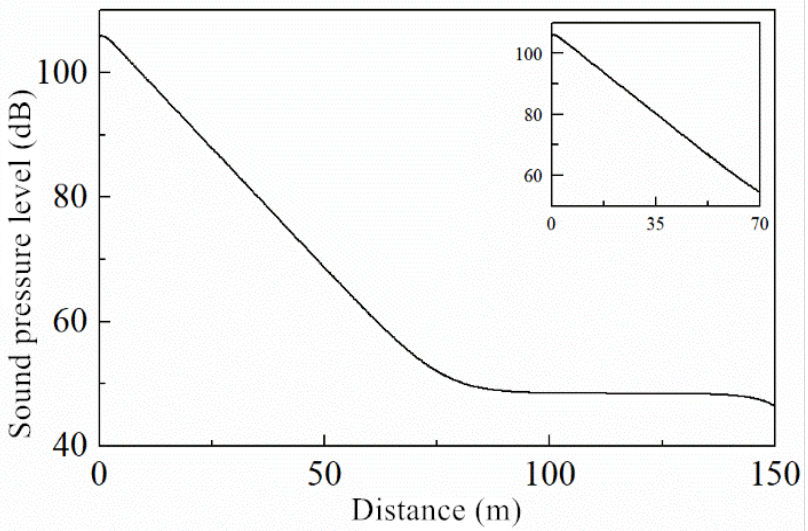

Fig.3. Change of sound pressure attenuation

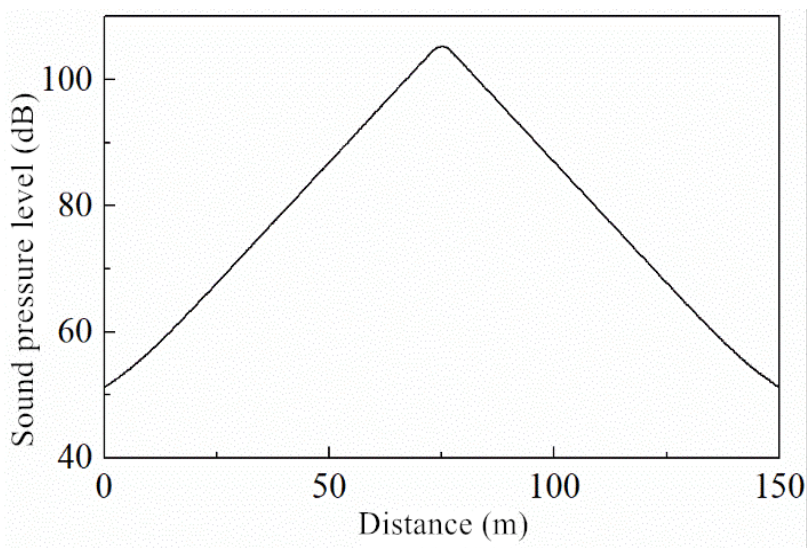

Fig.4. Change of sound pressure attenuation

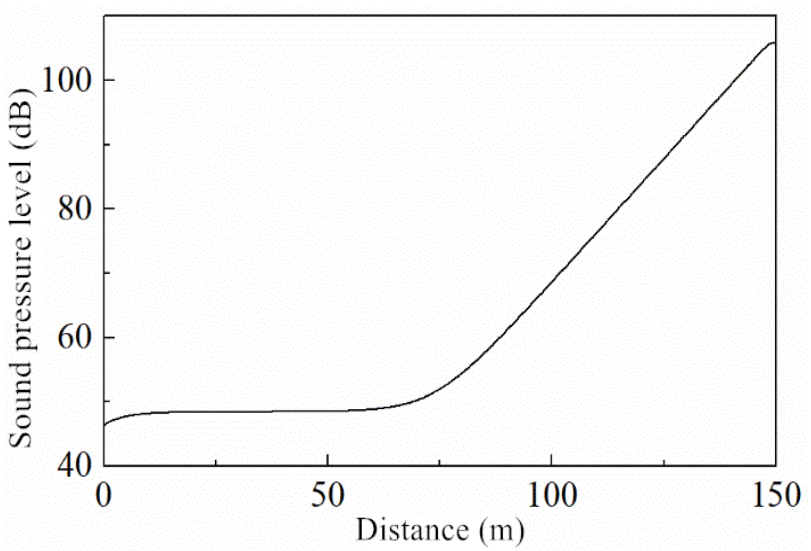

Fig.5. Change of sound pressure attenuation

When the sound source was localized at the rightmost end of the roadway goaf and the receiving point at the axial position of the sound source along the roadway center, the attenuation curves of the SPL with the increasing distance from the receiving point to the sound source are presented in Figure 5. The SPL also reached the maximum value at the exciting position. With the exciting point taken as the center, the SPL decreased from the initial $105.7 \mathrm{~dB}$ to $54.5 \mathrm{~dB}$ within the $70-\mathrm{m}$ range from the sound source, with an attenuation ratio of $48.4 \%$. The variation trend was rightly opposite the SPL attenuation with the increasing distance from the receiving point to the sound source in the roadway under the left-end excitation condition.

The comparison of the SPL attenuations under the leftend, middle, and right-end excitations with the increase in distance from the receiving point to the sound source in the roadway indicates that the SPL attenuation was unrelated to 
the point of action of the exciting position. The SPL attenuation under fixed excitation was identical, and the SPL distributions under the three circumstances are displayed in Figure 6.

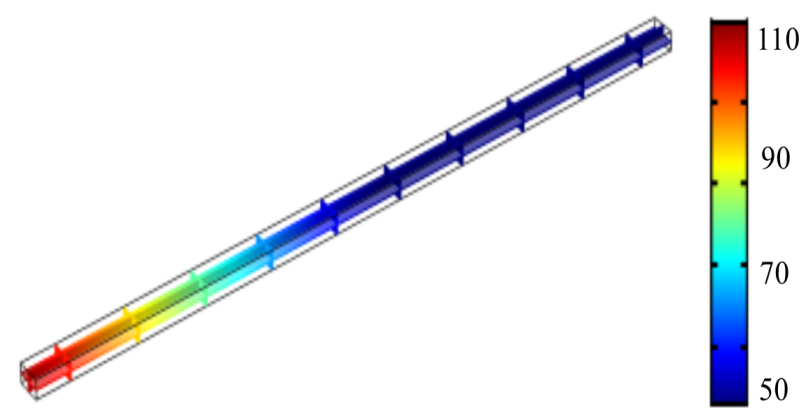

(a) Distribution map of sound pressure field in roadway under left end excitation

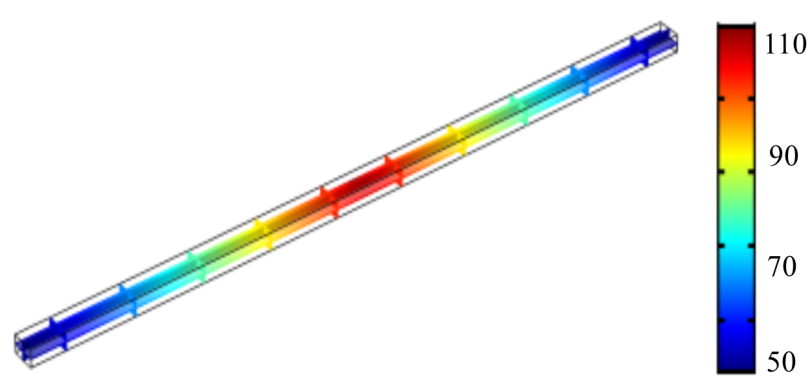

(b) Distribution map of sound pressure field in roadway under middle excitation

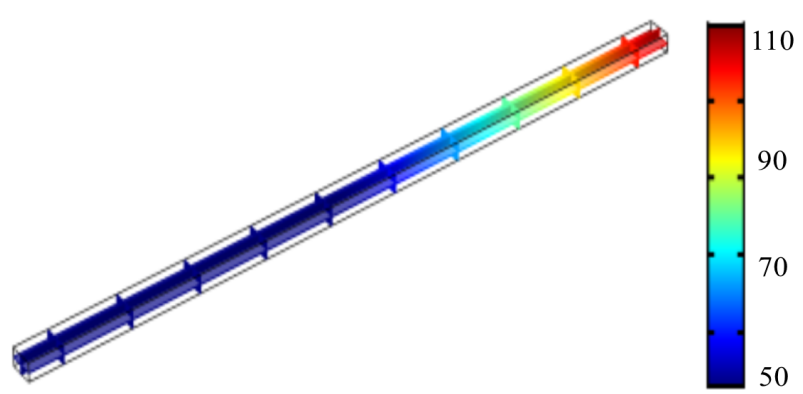

(c) Distribution map of sound pressure field in roadway under right end excitation

Fig. 6. Distribution map of sound pressure field in roadway

\subsection{Comparative analysis of measured data with simulation results}

The numerical comparison chart between the field measured values and the simulation results of noise situation in the fully mechanized mining face are shown in Figure 7. The measured SPL values of underground noise are consistent with the numerically simulated values. When the coal cutter was located at the leftmost end, the SPL of noise gradually declined as the receiving point became increasingly far from the sound source and exhibited a fast linear attenuation within the first $75-\mathrm{m}$ range. When the receiving point was 75-140 $\mathrm{m}$ away from the sound source, the sound energy was unchanged in the roadway, always in a steady linear attenuation state. As the receiving point approached the exit of the end face, the sound energy experienced a minor attenuation, the measured data conformed to the noise propagation and attenuation laws in long acoustic space (i.e., the roadway), the simulated values were also basically consistent with the measured values, and the maximum difference between the theoretical and measured values was 3-4 dB.
The main reasons for these findings are as follows. 1) The coal-rock wall surface is nonuniform in an actual fully mechanized mining roadway, and its geometrical shape is slightly different from the ideal model established in this study. 2) An actual fully mechanized mining roadway is of certain curvature and slope, and the theoretically computable data is established based on the cross-section perpendicular to the noise source along the longitudinal length direction of the roadway. 3) The disturbance from other mechanical and environmental noise is unavoidable in the actual measurement process of coal mines.

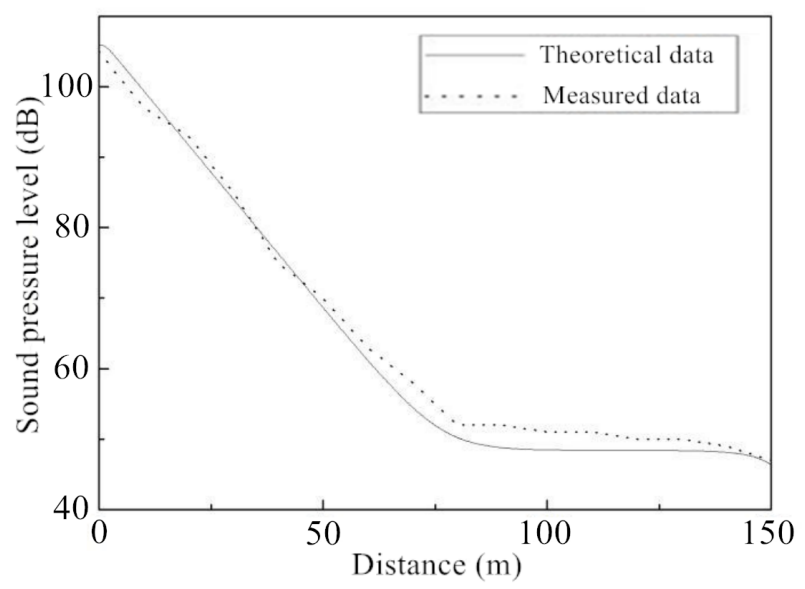

Fig.7. Comparison diagram of actual measurement and theoretical calculation

\section{Conclusion}

With respect to the influencing factors of underground noise propagation and the action mechanism, laboratory simulation tests and the collection of field investigation data from a coal mine were combined in this study. A mathematical model was constructed to simulate the noise field in the underground fully mechanized mining face in this coal mine via COMSOL Multiphysics, the scope and law of underground noise propagation at different sound source positions were analyzed, and the influence of sound source position on noise propagation and attenuation was determined. Finally, the simulation results were compared with the measured weighted SPL values of field noise, and the following conclusions were drawn:

(1) Directing at the parameters and physical quantities, which cannot be easily measured directly or can be directly measured but with great risks during the operation under noisy environment in a fully mechanized mining face, the noise field in the mining face was simulated using the COMSOL software to acquire the noise field distribution laws, compare with the measured weighted SPL values of underground noise, and verify the reliability of the proposed method.

(2) COMSOL can be used to accurately simulate the changes in noise level, propagation laws, and attenuation characteristics of the noise field in different areas of the underground fully mechanized coal mining face. The SPL attenuation of noise at the receiving point in the underground roadway of this fully mechanized mining face under different distances shows a linear symmetric trend.

(3) When the receiving point is near the sound source in the entire roadway space, the SPL attenuates rapidly, approximating a linear attenuation. When the receiving point is at a certain distance from the roadway exit, the sound 
energy is unchanged in the roadway. As the receiving point approaches the exit of the end face, the SPL experiences a minor attenuation again.

When the FEM is used to simulate the noise field in a fully mechanized mining face, complicated environmental problems can be avoided during data acquisition while providing a truly effective verification method for the experiments and more ideas and methods for noise control study, thus providing effective data support for exploring the noise propagation and attenuation laws in fully mechanized mining faces.

The findings of this study can be a powerful technical guarantee of reducing human error-induced accidents and occupational diseases and serve as a good guide in the safety management of coal mines. However, underground noise propagation and attenuation laws are influenced by various factors. Thus, the influencing factors require further investigation to lay a more solid foundation for follow-up studies.

\section{Acknowledgments}

This study was funded by the Joint Funds of the National Natural Science Foundation of China (No. U1904210), General Program of National Natural Science Foundation of China (No. 51774120) and Science and Technology Planning Project of Henan Province in China (202102310456) and Postdoctoral Research Fund of Anyang Institute of Technology (No. BHJ2021002) and Doctoral research fund of Anyang Institute of Technology (No. BSJ2020002) and Science and Technology Planning Project of Anyang in Henan Province (No. 2021C01SF042).

This is an Open Access article distributed under the terms of the Creative Commons Attribution License.

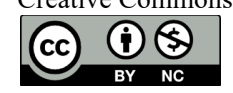

\section{References}

1. Cheng, J.Z., Wang, Y.B. "The comprehensive mechanized coal mining technology with good safety and efficiency at high working face". Shandong Coal science and Technology, (04), 2011, pp.142144.

2. Volo, M.D., Livi, R. "The influence of noise on synchronous dynamics in a diluted neural network". Chaos Solitons \& Fractals the Interdisciplinary, 57, 2013, pp.54-61.

3. Li, J., Qin, Y., Yang, L. "A simulation experiment study to examine the effects of noise on miners 'safety behavior in underground coal mines". BMC Public Health, 21(1), 2021, pp. 324 -336.

4. Thomas, C.G., Harshman, R.A., Menon R.S. "Noise reduction in bold-based FMRI using component analysis". Neuroimage, 17(3), 2002, pp.1521-1537.

5. Holmer, C.I. "Approach to interior noise control. I-Damped trim panels". Journal of Aircraft, 22(7), 2015, pp.618-623.

6. Akl, W., Baz, A. "Active vibration and noise control using smart foam". Journal of Vibration \& Control, 12(11), 2016, pp. 11731203.

7. Bunn, F., Zannin, P.H. "Urban planning-simulation of noise control measures". Noise Control Engineering Journal, 63(1), 2015, pp. 110 .

8. Ang, L.Y.L., Koh, Y. K., Lee, H. P. “Acoustic metamaterials: A potential for cabin noise control in automobiles and armored vehicles". International Journal of Applied Mechanics, 08(5), 2016, pp. 1650072.

9. Vemuri, S. H. S., Liu, X., Zang, B. Azarpeyvand, M. "On the use of leading-edge serrations for noise control in a tandem airfoil configuration". Physics of Fluids, 32(7), 2020, pp. 077102.

10. Mamontov, O., Stytsenko, T. "Development of a method for optimization calculation of a group of sound-insulating panels for air born noise protection". Eastern-European Journal of Enterprise Technologies, 3(10), 2019, pp. 32-38.

11. Sun, G., Li, M., Lim, T.C. "Enhanced filtered-x least mean Mestimate algorithm for active impulsive noise control". Applied Acoustics, 90(4), 2015, pp. 31-41.

12. Aslam, M.S., Raja, M. A. Z. "A new adaptive strategy to improve online secondary path modeling in active noise control systems using fractional signal processing approach". Signal Processing, SI (107), 2015, pp. 433-443.
13. Sun, H., Abhayapala, T.D. Samarasinghe, P.N., "A realistic multiple circular array system for active noise control over 3D space". IEEE/ACM Transactions on Audio, Speech, and Language Processing, 28, 2020, pp.3041-3052.

14. Yu, N., Li, Z.X., Wu, Y.F., Feng, R.J. "Quantum-behaved particle swarm optimization-based active noise control system with timing varying path". Journal of Low Frequency Noise Vibration and Active Control, 445, 2020, pp. 132-143.

15. Ahmed, S., Tufail, M., Rehan, M. “A novel approach for improved noise reduction performance in feed-forward active noise control systems with (loudspeaker) saturation non-linearity in the secondary path". IEEE/ACM Transactions on Audio, Speech, and Language Processing, 29, 2021, pp. 187-197.

16. Kim, J.W., Yang, S., Chung, I. "The effect of earplug training on noise protection". Annals of Occupational and Environmental Medicine, 31(3), 2019, pp. 1-6.

17. Ryu, J. K., Jin, Y. J. "Influence of noise sensitivity on annoyance of indoor and outdoor noises in residential buildings". Applied Acoustics, 72(6), 2011, pp.336-340.

18. Latos, M., Stankiewicz, K. "Studies on the effectiveness of noise protection for an enclosed industrial area using global active noise reduction systems". Journal of Low Frequency Noise Vibration and Active Control, 34(1), 2015, pp. 9-20.

19. Butorina, M., Oleinikov, A., Kuklin, D. "Classification of railway lines by noise emission for noise protection design". Akustika, 32, 2019, pp. 231-237.

20. Susanto, A., Setyawan, D.O., Setiabudi, F., et al. "GIS-based mapping of noise from mechanized minerals ore processing industry". Noise Mapping, 8(1), 2021, pp.1-15.

21. Anatoliy, P. S., Aleksandr, N. S., Svetlana, A.Y., Sergey V. G. "Noise protection of agricultural workers with cellular structures". Mordovia University Bulletin, 27(2), 2017, pp. 215-223.

22. Holt, A.G., Kuhl, A., Braun, R.D., Altschuler, R. "The rat as a model for studying noise injury and otoprotection". The Journal of the Acoustical Society of America, 146(5), 2019, pp.3681-3691.

23. Bin, W., Renyaun, F., Dasen, Z. "Study on acoustic character of active noise attenuation in ducts I theoretical analysis". Journal of the Beijing University of Technology. (4), 2003, pp. 411-413. 